\title{
Isolated Intracranial Rosai Dorfman Disease: A Rare Presentation with Diagnostic Mimickers
}

\author{
Ritika Singh $^{1 *}$, Milap Shah ${ }^{1}$, Anandh Balasubramaniam ${ }^{2}$, B.J Rajesh ${ }^{2}$ \\ ${ }^{1}$ Department of Pathology, Yashoda Hospital, Secunderabad, Telangana, India. \\ ${ }^{2}$ Department of Neurosurgery, Yashoda Hospital, Secunderabad, Telangana, India
}

\begin{abstract}
Rosai-Dorfman Disease (RDD) is an idiopathic lymphohisticyocytic disorder characterized clinically by massive painless cervical lymphadenopathy, fever and weight loss. Extranodal involvement has been recognized. Central nervous system (CNS) manifestations are rare. Intracranial localization of the disease without involvement of other sites is very rare and needs to be differentiated from various mimicking lesions especially meningioma. A radiological and histological confirmation followed by complete resection of the lesion is the treatment of choice.
\end{abstract}

Keywords: Rosai Dorfman Disease, Histiocytes, Emperipolesis, Dural, Meningioma.

\section{Introduction}

Sinus histiocytosis with massive lymphadenopathy, also known as Rosai-Dorfman

disease (RDD) was first described by Rosai and Dorfman in 1969. ${ }^{[1]}$ It presents as a massively enlarged, nontender cervical lymphadenopathy, accompanied by fever, leukocytosis, polyclonal hypergammaglobulinemia, anemia,and increased erythrocyte sedimentation rate. ${ }^{[2]} \mathrm{In}$ approximately $43 \%$ of cases, RDD manifests at extranodal sites, affecting the head and neck, sinuses, bones, respiratory tract, endocrine glands, and skin ${ }^{2}$. However even then associated lymphadenopathy may be present. Isolated central nervous system involvement is extremely rare with less than 40 cases reported. ${ }^{[3]}$

This report documents a 38-year-old man who presented with complaints of heachache and a decrease in vision and a dura-based suprasellar mass on radiology.

\section{Case report}

A 38 year old male presented with complaints of decrease in vision in both the eyes for the past six months with the reduction more in right eye than left. He also complained of headache which started one month back. Patient was taken up for a radiological evaluation.

Radiology : MRI brain revealed an intracranial suprasellar lesion that was homogeneously isointense relative to the gray matter on T1-weighted with avid homogeneous enhancement on contrast-enhanced T1-weighted image. (Figure 1)

Radiologically suggested differential diagnosis were meningioma or a granulomatous space occupying lesion.
Patient was taken up for surgery, the lesion was resected and the tissue was sent for histopathological examination.

Histopathology: Microscopic examination showed enlarged histiocytes admixed in a dense lymphoplasmacytic infitrate. Some of these histiocytes exhibited presence of intact plasma cells and lymphocytes in their cytoplasm consistent with emperipolesis. (Figure 2A, 2B)

Immunohistochemical studies were performed wherein the histiocytes were found to exhibit immunoreactivity for S100.(Figure 2C) They were negative for expression of CD 1a, CD 30, CD 15, CK.(Figure 2D).

Based on the above mentioned histological and immunohistochemical findings a final diagnosis of Rosai Dorfman disease was rendered.

Post operatively, ultrasound imaging of neck region along with abdomen and pelvis was done which did not show presence of any secondary lesion or lymphadenopathy.

\section{Discussion}

Extranodal RDD accounts for about $40 \%$ of cases, and usually involves the, skin, upper respiratory tract, orbits. RDD generally presents at around 20.6 years of age, but there is a varied age distribution ${ }^{[2]}$.

The exact cause of RDD remains controversial; however role played by immune (autoimmune dysfunction) and infectious factors (Human papillomavirus-6, Epstein-Barr virus, Brucella, and Cytomegalovirus ) has been suggested. ${ }^{[4]}$

Neurological involvement is rare, accounting for less than $5 \%$ of cases ${ }^{[2,5]}$. Intracranial RDD generally presents as a 


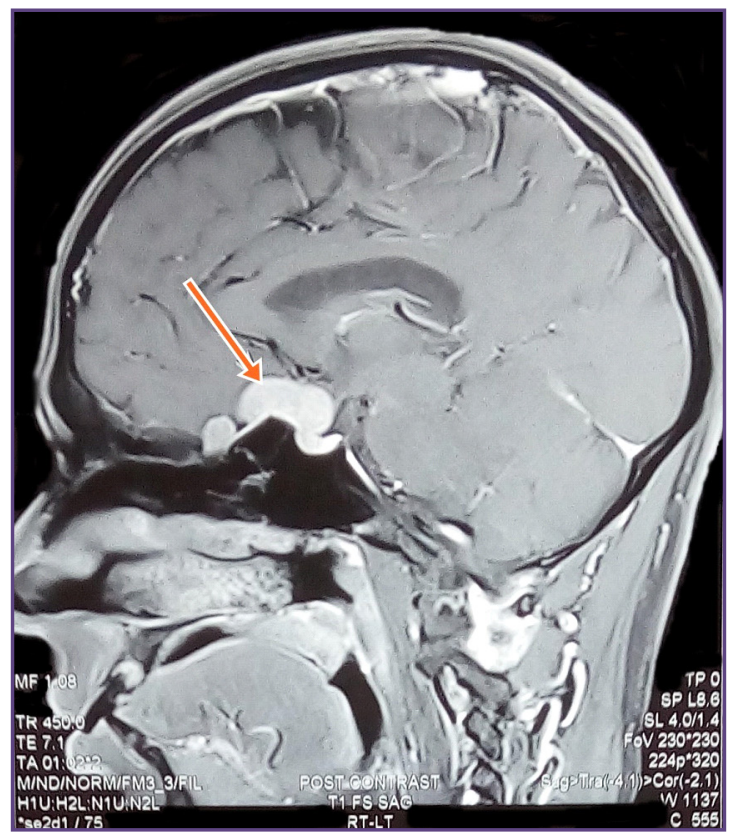

Fig. 1: Contrast-enhanced MR image showing a homogeneously enhanced suprasellar mass as indicated by arrow.

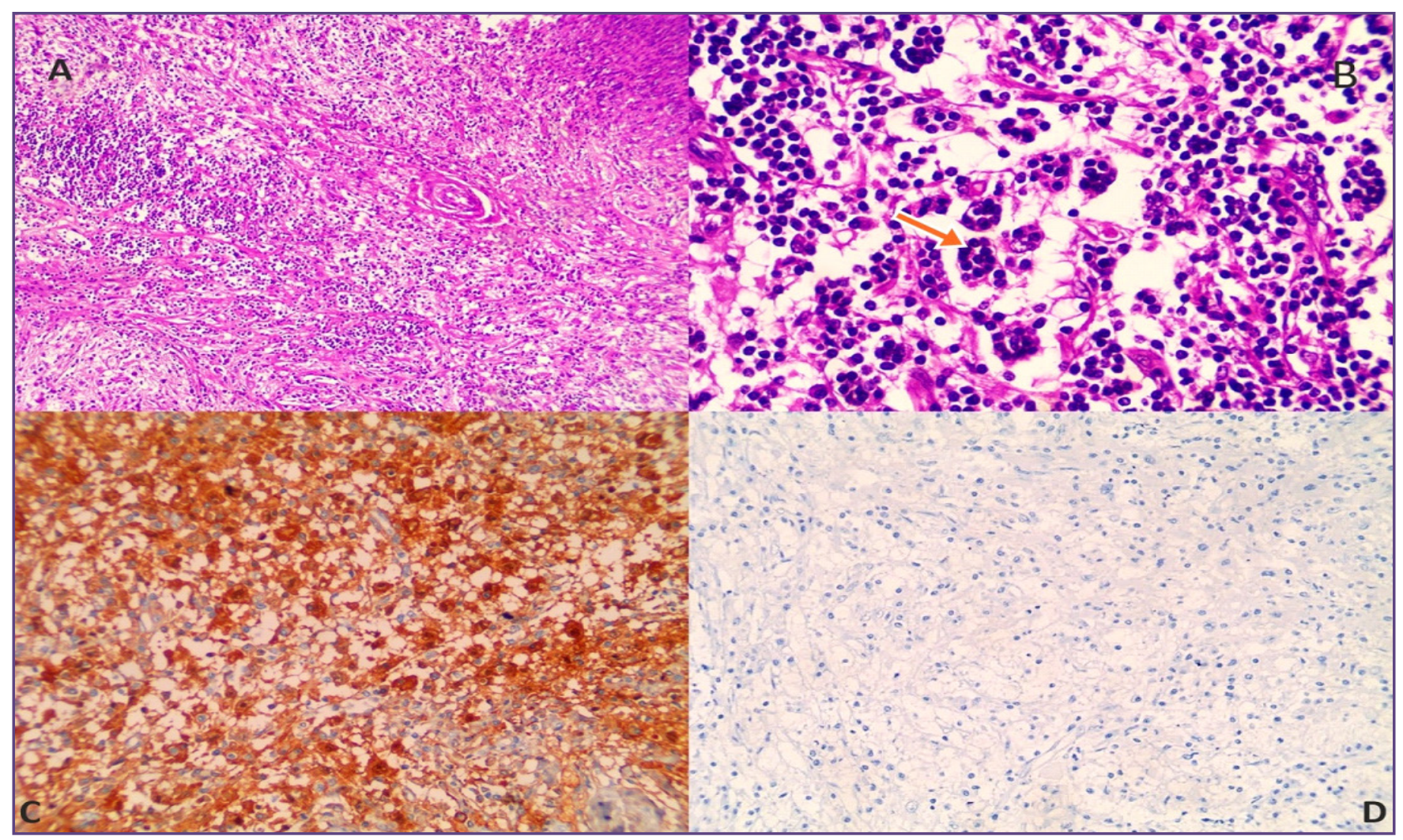

Fig. 2: A. Large histiocytes present in a dense lymphoplasmacytic infiltrate (haematoxylin and eosin, $\times 100$ ). B. Histiocytes show emperipolesis () (haematoxylin and eosin, $\times 400$ ) C. Histiocytes show immunopositivity for S100 but are; D. Negative for CD1a(avidin biotin complex immunoperoxidase method $\times 400$ ). 
solitary dural-based lesion but can involve multiple other intracranial sites. It can also present as intraparenchymal, intraventricular and cerebellar lesion. ${ }^{[6]}$ Of these isolated intracranial suprasellar involvement is extremely rare. ${ }^{[5]}$ In cases of intracranial involvement, the patients become symptomatic in fourth to fifth decade with a strong male preponderance. ${ }^{[7]}$

Clinical presentation usually seen in patients with intracranial involvement is headache, seizures, dysphasia, cranial nerve deficit, progressive loss of vision, hemiparesis and endocrine dysfunction depending on the location of the lesion. ${ }^{[8]}$

On imaging, many other lesions are found to mimick intracranial RDD. Hence the role of histological and immnohistochemical evaluation becomes vital.

These radiologic mimickers include meningioma, Langerhans cell histiocytosis, granulomatous diseases, plasmacytoma, Hodgkin's disease, plasma cell granulomas and metastasis. ${ }^{[6]}$ These are histologically differentiated as described below.

Intracranial RDD needs to be differentiated from lymphoplasmacytic meningioma in which a dense chronic inflammatory reaction, may be confused with infiltrates of the intracranial RDD. Identification of meningioma histology along with immunohistological positivity for epithelial membrane antigen helps in distinguishing the two. ${ }^{[9]}$

Langerhans histiocytosis often presents as small dural nodules and histologically may give a xanthomatous appearance. The two can be differentiated by differences in their nuclear features such as, the nuclei of Rosai-Dorfman histiocytes lack longitudinal nuclear grooves characteristic of Langerhans cells and often show emperipolesis. Also, the inflammatory infiltrate of Langerhans histocytosis contain eosinophils, which are not seen in cellular infiltrates of RDD. Even though on immunohistochemistry RDD and Langerhans' cell histiocytosis show S100 positivity, CD1a is usually negative in RDD. ${ }^{[10]}$

RDD-CNS may appear as Nodular sclerosing variant of Hodgkin's lymphoma due to the presence of fibrosis. Mononuclear Reed Sternberg (RS)cells variants, particularly lacunar cells may mimic RDD histiocytes. Classic RS cells and variants, lack emperipolesis and are S100 negative and show typically positivity for CD15 and CD30. ${ }^{[9]}$

Plasma cell granuloma may present as, dura-based inflammatory mass with associated fibrosis. Histologically these lesions do not show histiocytic cells. Hence histological and immunohistochemical S100 positivity in Intracranial RDD helps in distinguishing the two. ${ }^{[10]}$ Plasmacytoma is rare but well documented intracranially the lesions are attached to the dura and radiographically resemble meningioma. They can be immunohistochemically distinguished from RDD by the polyclonal nature of infiltrates in RDD. [ 9]

Management and treatment includes complete resection of the lesion. along with adjuvant therapy such as irradiation, chemotherapy and steroids to prevent disease recurrence. ${ }^{[6]}$

\section{Conclusion}

Although isolated intracranial Rosai-Dorfman disease is a rare diagnosis it should be considered in the differential diagnoses of dural-based mass and also intraparenchymal lesions of the CNS. Imaging studies help in detecting these lesions, but a definite diagnosis relies on the histological pattern and immunohistochemical characterization of the lesions.

\section{Abbreviations and Symbols}

Rosai Dorfman Disease: RDD

Central nervous system:CNS

Reed Sternberg cell: RS cell

\section{References}

1. Rosai J, Dorfman RF. Sinus histiocytosis with massive lymphadenopathy. A newly recognized benign clinicopathological entity. Arch Pathol. 1969; 87: 63-70.

2. Foucar E, Rosai J, Dorfman R. Sinus histiocytosis with massive lymphadenopathy (Rosai-Dorfman disease): review of the entity. Semin Diagn Pathol. 1990; 7: 19-73.

3. Kumar R, Garg K, Dash C, Sharma MC. Rosai-Dorfman disease:An unusual isolated cavernous sinus lesion. Neurol India. 2016;64:1331-2

4. Hong CS, Starke RM, Hays MA, Mandell JW, et al. Redefining the Prevalence of Dural Involvement in RosaiDorfman Disease of the Central Nervous System.World Neurosurg. $2016 ; 90: 702$.

5. Wan S, Teng X, Zhan R, Yu J, Gu J, Zhang K. Isolated intracranial Rosai-Dorfman disease mimicking suprasellar meningioma: case report with review of the literature. J Int Med Re. 2008;36:1134-9.

6. Raslan OA, Schellingerhout D, Fuller GN. Rosai-Dorfman disease in neuroradiology: imaging findings in a series of 10 patients. AJR Am J Roentgenol. 2011; 196: 187-93.

7. Deodhare SS, Ang LC, Bilbao JM. Isolated intracranial involvement in Rosai-Dorfman disease: a report of two cases and review of the literature. Arch Pathol Lab Med. 1998; 122: 161-5. 
8. Symss NP, Cugati G, Vasudevan MC,Ramamurthi R, Pande A. Intracranial Rosai Dorfman Disease: report of three cases and literature review. Asian J Neurosurg. 2010;5:19-30.

9. Andriko JA, Morrison A, Colegial CH, Davis BJ, Jones
RV. Rosai-Dorfman disease isolated to the central nervous system: a report of 11 cases. Mod Pathol. 2001;14:172-8.

10. Prayson A R, Rowe JJ Dural-based Rosai-Dorfman disease: Differential diagnostic considerations. J of Clinical Neuroscience. 2014;21:1872-3.

*Corresponding author:

Dr. Ritika Singh, 301, A-23, Sanskruti Township Pocharam, Hyderabad-500088. INDIA

Phone: +91 9958492397

Email: ritika84singh@gmail.com

Financial or other Competing Interests: None. 